\title{
Chapter 10 \\ Transgenerational Effects on Calf Spermatogenesis and Metabolome Associated with Paternal Exposure to the Fukushima Nuclear Power Plant Accident
}

\author{
Banri Suzuki, Shigefumi Tanaka, Kohichi Nishikawa, Chikako Yoshida, \\ Takahisa Yamada, Yasuyuki Abe, Tomokazu Fukuda, Jin Kobayashi, \\ Gohei Hayashi, Masatoshi Suzuki, Yusuke Urushihara, Kazuma Koarai, \\ Yasushi Kino, Tsutomu Sekine, Atsushi Takahasi, Toshihiro Shimizu, \\ Hisashi Shinoda, Kazuki Saito, Emiko Isogai, Koh Kawasumi, \\ Satoshi Sugimura, Hideaki Yamashiro, and Manabu Fukumoto
}

\begin{abstract}
The Fukushima nuclear power plant (FNPP) accident raised worldwide attention to the health risk of radiation exposure and to its potential transgenerational effects. Here, we analysed transgenerational effects on calf spermatogenesis and on blood plasma metabolome in order to detect alterations associated with paternal exposure to low-dose-rate (LDR) radiation. Sperm was collected from a bull exposed to radiation for 2 years abandoned in the ex-evacuation zone of the FNPP accident (the abandoned bull) and was used for artificial insemination (AI) into a non-radiocontaminated cow. Haematoxylin and eosin stained sections of the testis of a 13-month-old calf revealed spermatogonia, spermatocytes, spermatids, and sperm in normal morphology. Nuclear and acrosomal morphology of sperm
\end{abstract}

B. Suzuki $\cdot$ S. Tanaka $\cdot$ K. Nishikawa $\cdot$ C. Yoshida $\cdot$ T. Yamada $\cdot$ H. Yamashiro $(\bowtie)$

Graduate School of Science and Technology, Niigata University, Niigata, Japan

e-mail: hyamashiro@agr.niigata-u.ac.jp

Y. Abe

Department of Life Science, Prefectural University of Hiroshima, Hiroshima, Japan

T. Fukuda

Graduate School of Agricultural Sciences, Iwate University, Morioka, Japan

J. Kobayashi

School of Food, Agricultural, and Environmental Sciences, Miyagi University, Sendai, Japan

G. Hayashi · M. Suzuki

Institute of Development, Aging and Cancer, Tohoku University, Sendai, Japan 
was generally normal. Metabolomic profiling of plasma using capillary electrophoresis-mass spectrometry resulted in 104 peaks of candidate compounds suggestive of paternal exposure. A calf was delivered by AI using sperm from the abandoned bull. Regarding glycolysis, the contents of nucleotide sugars tended to be lower in the delivered calf than in the control calf. Among energy carries, AMP and ATP showed different tendency between non-radiocontaminated and delivered calf. In conclusion, there were no apparent transgenerational effects on both spermatogenesis and blood plasma metabolome in a calf obtained by AI using sperm from the abandoned bull exposed to LDR in the ex-evacuation zone of the FNPP accident for about 2 years.

Keywords Calf · Fukushima Daiichi Nuclear Power Plant accident · Low-doserate $\cdot$ Metabolome $\cdot$ Spermatogenesis

\title{
10.1 Introduction
}

Following the Fukushima Daiichi Nuclear Power Plant (FNPP) accident, large amounts of radioactive substances, particularly volatile elements such as radioactive iodine $\left({ }^{131} \mathrm{I},{ }^{132} \mathrm{I}\right.$ and $\left.{ }^{133} \mathrm{I}\right)$, cesium $\left({ }^{134} \mathrm{Cs},{ }^{136} \mathrm{Cs}\right.$ and $\left.{ }^{137} \mathrm{Cs}\right)$, tellurium $\left({ }^{132} \mathrm{Te}\right)$, and inert gases (e.g., ${ }^{133} \mathrm{Xe}$ ), were released into the environment [1]. We have established an archive system composed of livestock and wild animals in a 20-km radius around FNPP, that is, the ex-evacuation zone of the FNPP accident [2-5]. This system provides critical information for the understanding of radioactive contamination, environmental pollution, biodistribution and metabolism, and the biological effects of internal and external exposure in association with dose evaluation. Recently, we

\author{
Y. Urushihara \\ Graduate School of Medicine, Tohoku University, Sendai, Japan \\ K. Koarai · Y. Kino · T. Sekine \\ Graduate School of Science, Tohoku University, Sendai, Japan \\ A. Takahasi $\cdot$ T. Shimizu $\cdot$ H. Shinoda \\ Graduate School of Dentistry, Tohoku University, Sendai, Japan \\ K. Saito $\cdot$ E. Isogai \\ Graduate School of Agricultural Sciences, Tohoku University, Sendai, Japan \\ K. Kawasumi \\ School of Veterinary Medicine, Nippon Veterinary and Life Science University, Tokyo, Japan \\ S. Sugimura \\ Institute of Agriculture, Tokyo University of Agriculture and Technology, Tokyo, Japan \\ M. Fukumoto \\ Institute of Development, Aging and Cancer, Tohoku University, Sendai, Japan \\ School of Medicine, Tokyo Medical University, Tokyo, Japan
}


reported organ-specific deposition of the individual radionuclide in abandoned cattle following the FNPP accident. Radioactive caesium $\left({ }^{134} \mathrm{Cs}\right.$ and $\left.{ }^{137} \mathrm{Cs}\right)$ is notably detected in all organs examined [6-8]. Further, we reported that spermatogenesis occurred normally in the reproductive organs of bulls and boars that were abandoned in the ex-evacuation zone, following chronic low-dose-rate (LDR) radiation $[9,10]$. However, to date there is no clear evidence whether transgenerational effects of radiation exposure in livestock animals associated with the FNPP accident exist or not.

Here, we analysed transgenerational effects on the calf spermatogenesis, and blood plasma metabolome using capillary electrophoresis-mass spectrometry (CE-TOFMS) with paternal exposure to chronic LDR radiation by staying for 2 years in the ex-evacuation zone.

\subsection{Materials and Methods}

\subsubsection{Ethics}

The Japanese government ordered Fukushima Prefecture to euthanize cattle in the ex-evacuation zone on May 12, 2011 to prevent radio-contaminated beef products from entering the human food chain. We collected organs and tissues from the euthanized cattle by the combined unit of veterinary doctors belonging to the Livestock Hygiene Service Center of Fukushima Prefecture and those belonging to the Ministry of Agriculture, Forestry and Fisheries, Japan. This study was approved by the Ethics Committee of Animal Experiments, Niigata University, Japan (Regulation No. 27-83-3).

\subsubsection{Animals}

We collected sperm form the testis of an euthanized Japanese black bull (the abandoned bull) at Tomioka Town, located $7 \mathrm{~km}$ south of FNPP, on February 28, 2013, as described previously [10]. Immediately after collection, the sperm was diluted with a Triladyl freezing extender containing egg yolk (Mini Tube, Germany); freezing protocol was performed as described previously [11]. On sampling date, ambient dose equivalent rate was $1.7 \mu \mathrm{Sv} / \mathrm{h}$ (using a $\mathrm{NaI}$ (Tl) Scintillation Survey Meter), and the time elapsed since the FNPP accident (March 11, 2011) was almost 2 years. Dose rate of both internal and external exposure to ${ }^{134} \mathrm{Cs}$ and ${ }^{137} \mathrm{Cs}$ was estimated according to a modified method as previously described [3]. 


\subsubsection{Artificial Insemination (AI)}

Using the sperm of the abandoned bull, a non-radiocontaminated recipient black cow underwent AI at the experimental farm of Niigata University. A male Japanese black calf (the delivered calf) was born on August 29, 2015 (approximately 10-month pregnancy period) from which the testis, caudae epididymides, sperm, and blood plasma were sampled on September 27, 2016 (at approximately 13 months old) after euthanasia. As non-radiocontamination control, blood plasma was also collected from a non-castrated male Japanese black calf at almost the same age as the delivered calf from Niigata Prefecture, at the same latitude as Fukushima but not affected by the FNPP accident. The relationship between each cattle and artificial insemination is shown in Fig. 10.1.

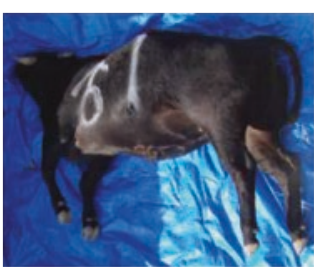

The abandoned bull

(Sperm collected on 2/28/2013)

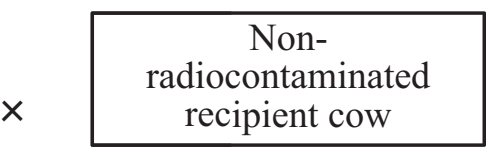

\section{Artificial insemination (AI)}

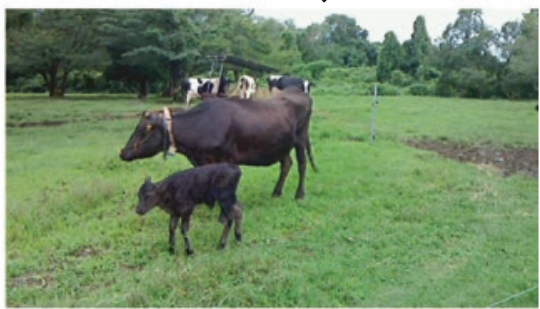

The delivered calf

(Born on 8/29/2015)

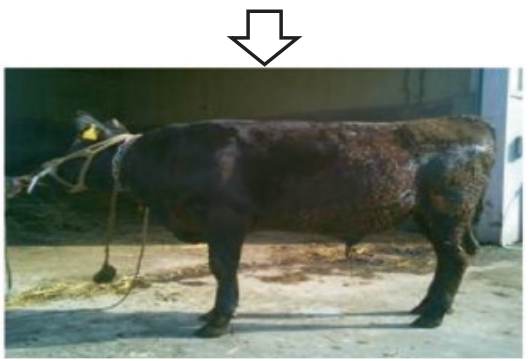

(Sampling on 9/27/2016)

Fig. 10.1 The delivered calf obtained by artificial insemination using frozen sperm from an abandoned bull in the ex-evacuation zone of the FNPP accident 


\subsubsection{Histological Analysis}

The delivered calf testis was fixed in Bouin's solution. Sections were stained with haematoxylin and eosin (HE) and analysed microscopically as previously described [12].

\subsubsection{Evaluation of Sperm Acrosomal Integrity}

The acrosomal integrity of the delivered calf sperm was assessed by staining with fluorescein isothiocyanate-conjugated peanut agglutinin (FITC-PNA; Wako, Japan) according to the procedure described previously [13].

\subsubsection{Measurement of Metabolites}

Metabolome analysis of blood plasma was carried out by a facility service, Human Metabolome Technologies Inc. (Yamagata, Japan), according to the conditions described elsewhere [14-16]. A capillary electrophoresis system, Agilent 6210 Time-of-Flight LC/MS (Agilent Technologies, Waldbronn, Germany), was used for capillary electrophoresis time-of-flight mass spectrometry (CE-TOF-MS). The spectrometer scanned from $\mathrm{m} / \mathrm{z} 50$ to 1,000 . Peak information included $\mathrm{m} / \mathrm{z}$, migration time (MT) and peak area. Peaks were obtained using an automatic integration software MasterHands (Keio University, Tsuruoka, Japan) [17]. Signal peaks corresponding to isotopomers, adduct ions and other product ions of known metabolites were excluded, and the remaining peaks were annotated with putative metabolites from the HMT metabolite database based on their $m / z$ and MTs values determined by TOF-MS. The tolerance range for peak annotation was configured at $\pm 0.5 \mathrm{~min}$ for MT and $\pm 10 \mathrm{ppm}$ for $\mathrm{m} / \mathrm{z}$. In addition, peak areas were normalised against those of the internal standards, and then the resultant relative area values were further normalised by the sample amount.

Hierarchical cluster analysis (HCA) and principal component analysis (PCA) were performed by our proprietary software, PeakStat and SampleStat, respectively. Detected metabolites were plotted on metabolic pathway maps using VANTED (Visualization and Analysis of Networks containing Experimental Data) software [18].

Compounds with a relative area ratio smaller than 0.66 or bigger than 1.5 were defined as having different levels between the delivered calf and the nonradiocontaminated control. 


\subsection{Results and Discussion}

Total dose-rate in the testis of the abandoned bull attributed to radioactive Cs was $33.9 \mu \mathrm{Gy} /$ day $(2.7 \mu \mathrm{Gy} /$ day for internal exposure and $31.2 \mu \mathrm{Gy} /$ day for external exposure).

Histology of the testis from the delivered calf at 13 months old revealed no remarkable changes in spermatogonia, spermatocytes, spermatids and sperm (Fig. 10.2). Sperm nuclear and acrosomal morphology was generally normal (Fig. 10.3). Abnormal sperm morphology, featuring partly absent acrosome, was observed at a rate of $7.0 \%$ (Table 10.1). These results show that, in the present study, paternal exposure to LDR radiation persistently did not affect the spermatogenesis process for calf.

CE-TOF-MS-mediated metabolic profiling of plasma resulted in 104 peaks of candidate compounds associated with exposure to chronic LDR radiation (hereafter "detected candiate" compounds). Among energy carries, AMP (ratio $=0.5$ ) and ATP (ratio = 1.6) showed different levels between the delivered calf and the nonradiocontaminated control, whereas ADP did not (Table 10.2; Fig.10.4). Detected candidate compounds associated with the energy supply system are shown in Table 10.3 and Fig. 10.3. Regarding glycolysis, the present study showed that contents of nucleotide sugars such as G1P, G6P, DHAP and 3-PG were lower in the
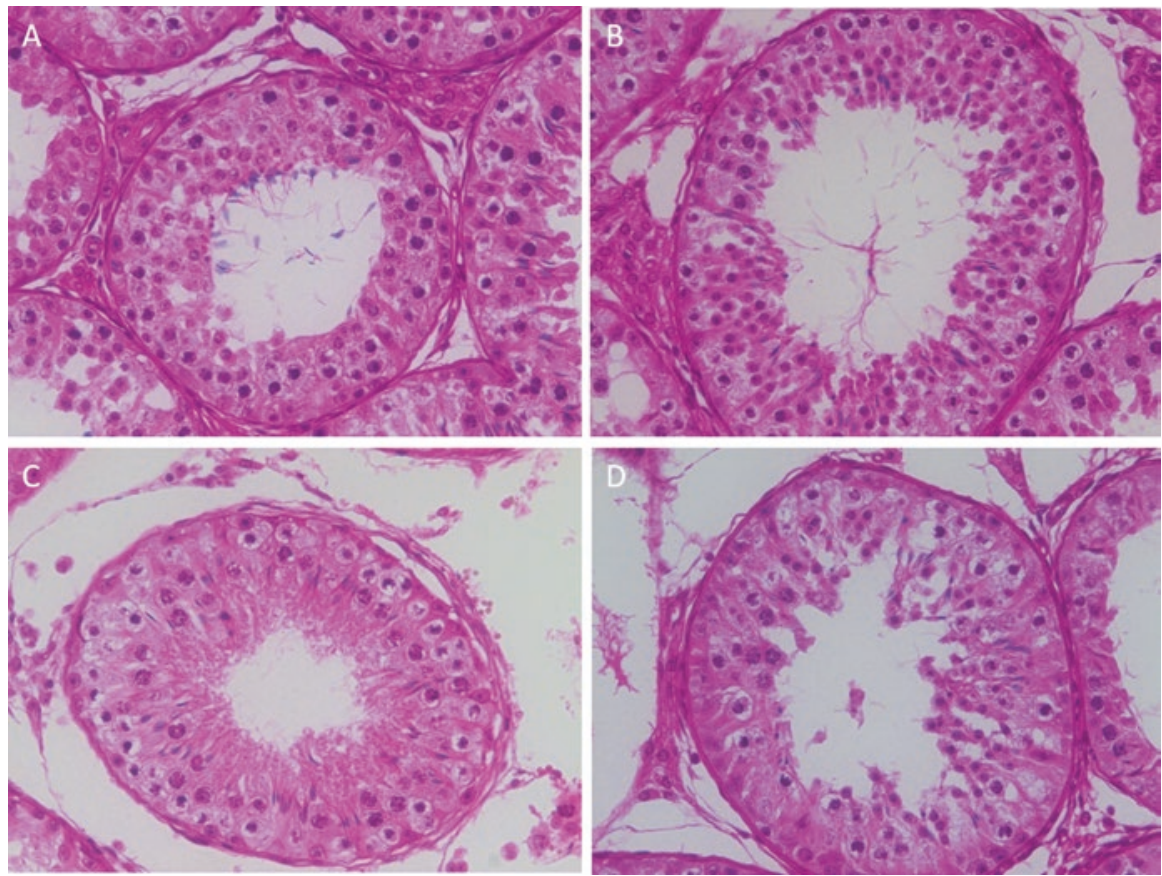

Fig. 10.2 Histological sections of the seminiferous tubules of the delivered calf testis. Scale bar, $100 \mu \mathrm{m}$ in $\mathbf{a}$ and $\mathbf{d}$; magnification, 200x 

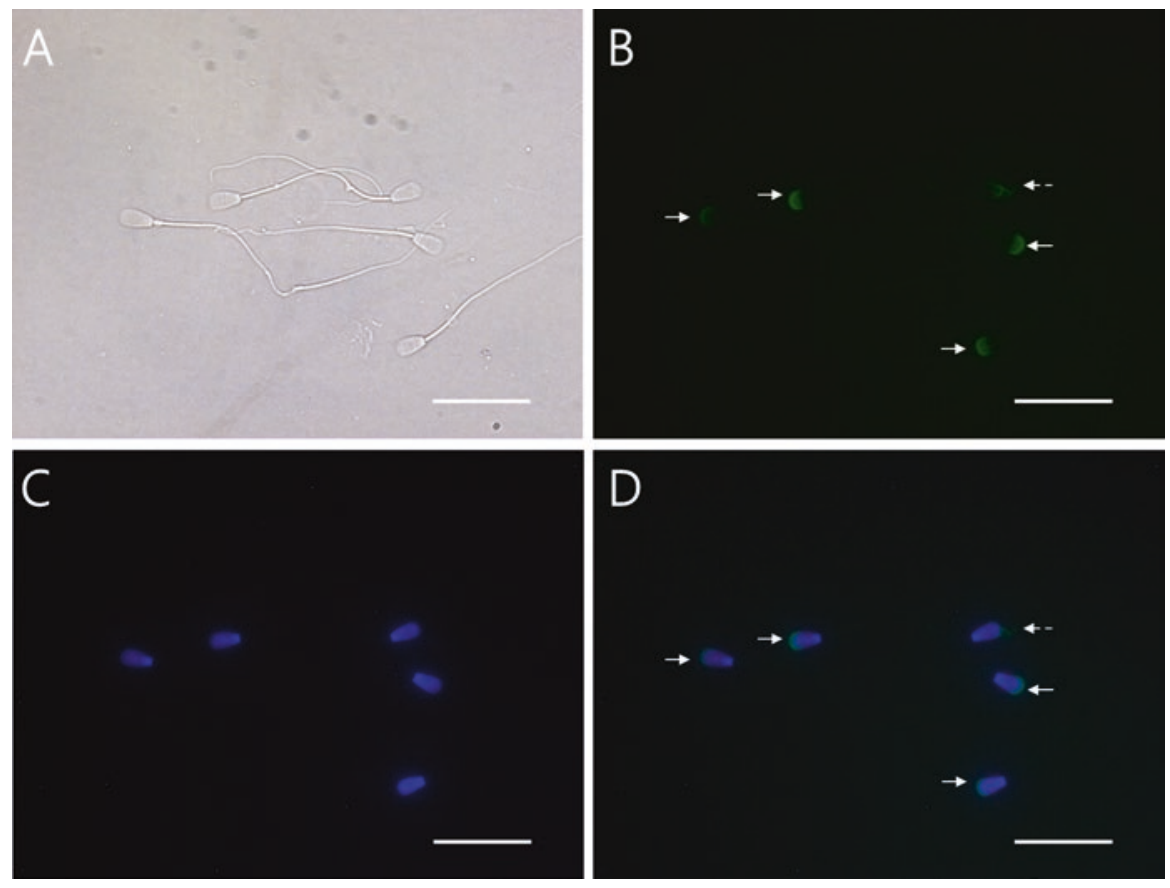

Fig. 10.3 Epididymal sperm nuclei and acrosomes of the delivered calf stained with $4^{\prime}, 6^{\prime}$-diamino2'-phenylindole (DAPI) and fluorescein isothiocyanate-conjugated peanut agglutinin (FITCPNA). (a) Phase contrast, (b) DAPI-stained, (c) FITC-PNA-stained and (d) DAPI+FITC-PNA-stained images. Scale bar, $50 \mu \mathrm{m}$; magnification, 400x

Table 10.1 Rates of sperm acrosome normal or abnormal morphology

\begin{tabular}{l|l|l|l}
\hline & \multicolumn{2}{|l|}{ Sperm acrosome morphology } & \multirow{2}{*}{ Total } \\
\cline { 2 - 4 } & Normal (\%) & Abnormal $^{\mathrm{a}}(\%)$ & 200 \\
\hline Number of sperm & $186(93.0)$ & $14(7.0)$ & 200 \\
\hline
\end{tabular}

${ }^{a}$ Abnormal sperm morphology, featuring partly absent acrosome

Table 10.2 Detected candidate energy-carrier compounds

\begin{tabular}{l|l|l|l}
\hline \multirow{2}{*}{ Energy carrier } & \multicolumn{2}{|l|}{ Relative area } & Comparative analysis $^{\mathrm{a}}$ \\
\cline { 2 - 4 } & Control calf & Delivered calf & Ratio \\
\hline ADP & $7.0 \mathrm{E}-04$ & $7.5 \mathrm{E}-04$ & 1.1 \\
\hline AMP & $4.5 \mathrm{E}-04$ & $2.1 \mathrm{E}-04$ & 0.5 \\
\hline ATP & $3.4 \mathrm{E}-04$ & $5.5 \mathrm{E}-04$ & 1.6 \\
\hline
\end{tabular}

${ }^{\mathrm{a}}$ Control relative area served as denominator

${ }^{\mathrm{b}}$ The delivered calf with paternal exposure to radiation $(n=1)$ 


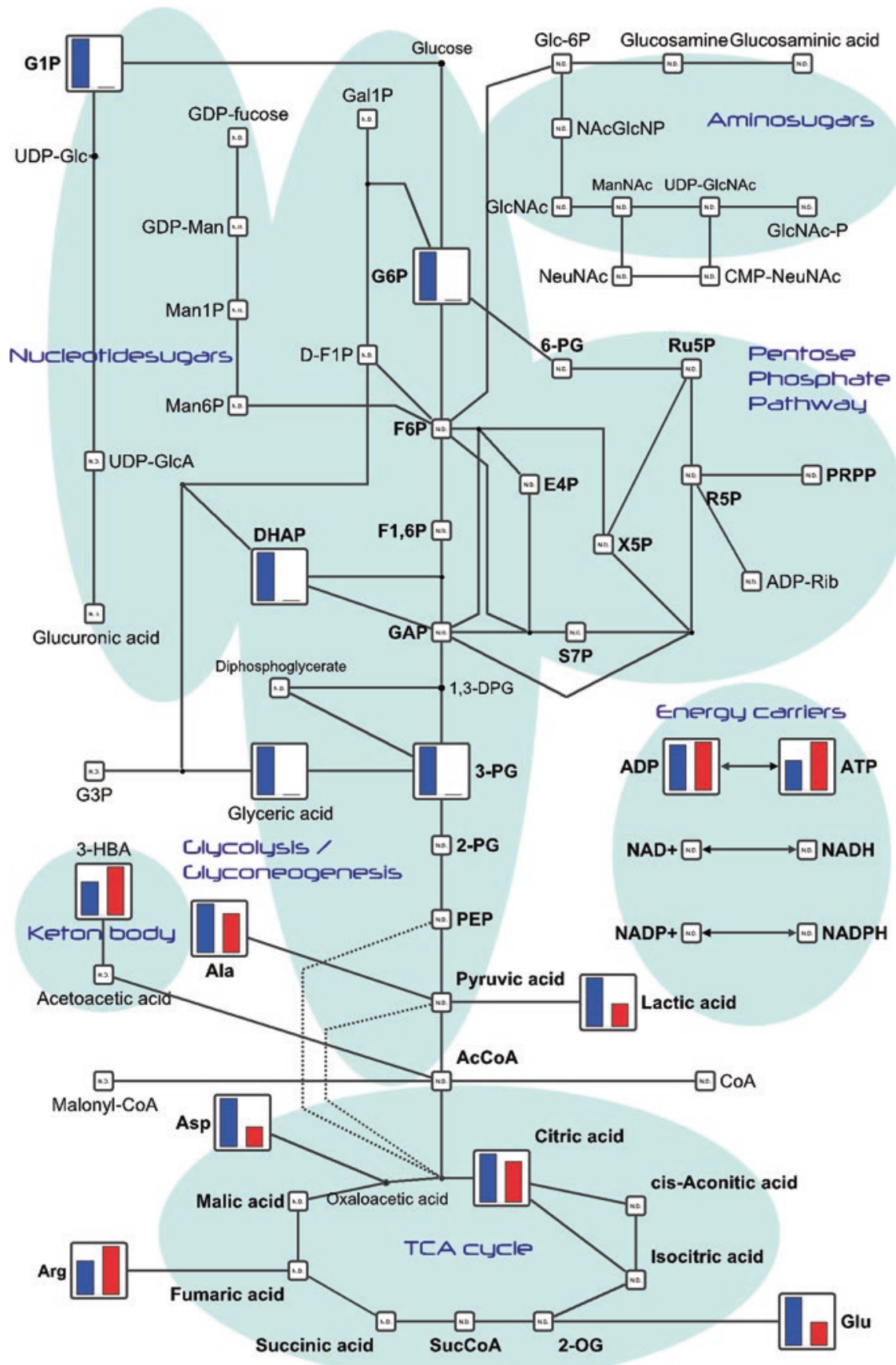

Fig. 10.4 Diagram of carbohydrate metabolism of the delivered calf (red) and the nonradiocontaminated control (blue). The control relative area was served as the denominator 
10 Transgenerational Effects on Calf Spermatogenesis and Metabolome Associated...

Table 10.3 Detected candidate compounds involved in carbohydrate metabolism

\begin{tabular}{l|l|l|l}
\hline \multirow{2}{*}{ Carbohydrate metabolite } & Relative area & ${\text { Comparative } \text { analysis }^{\mathrm{a}}}^{\mathrm{n}}$ \\
\cline { 2 - 4 } Control calf & Delivered calf & Ratio \\
\hline 3-Hydroxybutyric acid & $2.6 \mathrm{E}-02$ & $3.8 \mathrm{E}-02$ & 1.4 \\
\hline Citric acid & $2.9 \mathrm{E}-02$ & $2.5 \mathrm{E}-02$ & 0.8 \\
\hline Lactic acid & $1.1 \mathrm{E}-01$ & $5.0 \mathrm{E}-02$ & 0.5 \\
\hline
\end{tabular}

${ }^{a}$ Control relative area served as denominator

${ }^{\mathrm{b}}$ The delivered calf with paternal exposure to radiation $(n=1)$

Table 10.4 Detected candidate amino acids

\begin{tabular}{l|l|l|l}
\hline \multirow{2}{*}{ Amino acid } & \multicolumn{2}{l}{ Relative area } & Comparative analysis $^{\mathrm{a}}$ \\
\cline { 2 - 4 } Ala & Control calf & Delivered calf & Ratio \\
\hline Arg & $5.9 \mathrm{E}-02$ & $4.7 \mathrm{E}-02$ & 0.8 \\
\hline Asn & $1.9 \mathrm{E}-02$ & $2.7 \mathrm{E}-02$ & 1.4 \\
\hline Asp & $5.2 \mathrm{E}-03$ & $6.2 \mathrm{E}-03$ & 1.2 \\
\hline Cys & $3.2 \mathrm{E}-03$ & $1.3 \mathrm{E}-03$ & 0.4 \\
\hline Gln & N. D. & \multicolumn{2}{l}{} \\
\hline Glu & $4.8 \mathrm{E}-02$ & $7.1 \mathrm{E}-02$ & 1.5 \\
\hline Gly & $2.3 \mathrm{E}-02$ & $1.1 \mathrm{E}-02$ & 0.5 \\
\hline His & $5.0 \mathrm{E}-02$ & $4.9 \mathrm{E}-02$ & 1.0 \\
\hline Ile & $1.6 \mathrm{E}-02$ & $1.7 \mathrm{E}-02$ & 1.1 \\
\hline Leu & $5.4 \mathrm{E}-02$ & $6.3 \mathrm{E}-02$ & 1.2 \\
\hline Lys & N. D. & \multicolumn{1}{l}{} \\
\hline Met & $1.7 \mathrm{E}-02$ & $2.4 \mathrm{E}-02$ & 1.4 \\
\hline Phe & $7.8 \mathrm{E}-03$ & $6.7 \mathrm{E}-03$ & 0.9 \\
\hline Pro & $2.4 \mathrm{E}-02$ & $2.3 \mathrm{E}-02$ & 1.0 \\
\hline Ser & $2.9 \mathrm{E}-02$ & $2.5 \mathrm{E}-02$ & 0.9 \\
\hline Thr & $1.1 \mathrm{E}-02$ & $1.4 \mathrm{E}-02$ & 1.3 \\
\hline Trp & $2.0 \mathrm{E}-02$ & $1.7 \mathrm{E}-02$ & 0.8 \\
\hline Tyr & $1.1 \mathrm{E}-02$ & $1.3 \mathrm{E}-02$ & 1.1 \\
\hline Val & $1.5 \mathrm{E}-02$ & $1.5 \mathrm{E}-02$ & 1.0 \\
\hline & $1.0 \mathrm{E}-01$ & $1.1 \mathrm{E}-01$ & 1.1 \\
\hline
\end{tabular}

${ }^{a}$ Control relative area served as denominator

${ }^{b}$ Delivered calf associated with paternal exposure to radiation $(n=1)$

delivered calf than in the control calf. Lactic acid, a final product of glycolysis under an aerobic conditions, featured lower-level tendency in the delivered calf than in the control calf (ratio $=0.5)$. 3-Hydroxybutyrate $(3-\mathrm{HBA})$ is a ketone body used as an energy source, whereby it is converted to acetyl CoA and oxidised in the TCA cycle. 3 -HBA showed also no difference (ratio $=1.4$ ). Citric acid is a metabolite of the TCA cycle that showed no difference between the delivered calf and the control $($ ratio $=0.8)$. This indicates that, under anaerobic conditions, the energy use of the delivered calf has lower tendency than that of the non-radiocontamined calf.

Amino acids catabolized to simple intermediate and oxidised in the TCA cycle showed almost no difference between the delivered calf and the control (Table 10.4), 
with the exception of aspartic acid (Asp; ratio $=0.4)$, glutamine $(\mathrm{Gln}$; ratio $=1.5)$, and glutamine acid $(\mathrm{Glu}$; ratio $=0.5)$. Glu collects amino groups from most amino acids, including Asp, followed by conversion to $\alpha$-ketoglutarate. Since Gln is converted to Glu by glutaminase, it is possible that higher levels of Gln in the delivered calf, as compared with the control calf, serve to produce Glu, which in turn activates amino acid catabolism. These may also explain the higher ATP levels detected in the delivered calf.

Arg, Asp, citrulline, and ornithine participate in the urea cycle (Table 10.5 and Fig. 10.5), a pathway that eliminates ammonia outside the body by converting it to

Table 10.5 Detected candidate compounds involved in urea metabolism

\begin{tabular}{l|l|l|l}
\hline \multirow{2}{*}{ Urea metabolite } & \multicolumn{2}{|l|}{ Relative area } & Comparative analysis $^{\mathrm{a}}$ \\
\cline { 2 - 4 } & Control calf & Delivered calf & Ratio \\
\hline Citrulline & $1.6 \mathrm{E}-02$ & $1.4 \mathrm{E}-02$ & 0.9 \\
\hline Ornithine & $1.2 \mathrm{E}-02$ & $1.5 \mathrm{E}-02$ & 1.3 \\
\hline Urea & $3.8 \mathrm{E}-01$ & $5.2 \mathrm{E}-01$ & 1.4 \\
\hline
\end{tabular}

${ }^{\mathrm{a} C o n t r o l ~ r e l a t i v e ~ a r e a ~ s e r v e d ~ a s ~ d e n o m i n a t o r ~}$

${ }^{b}$ Delivered calf associated with paternal exposure to radiation $(n=1)$

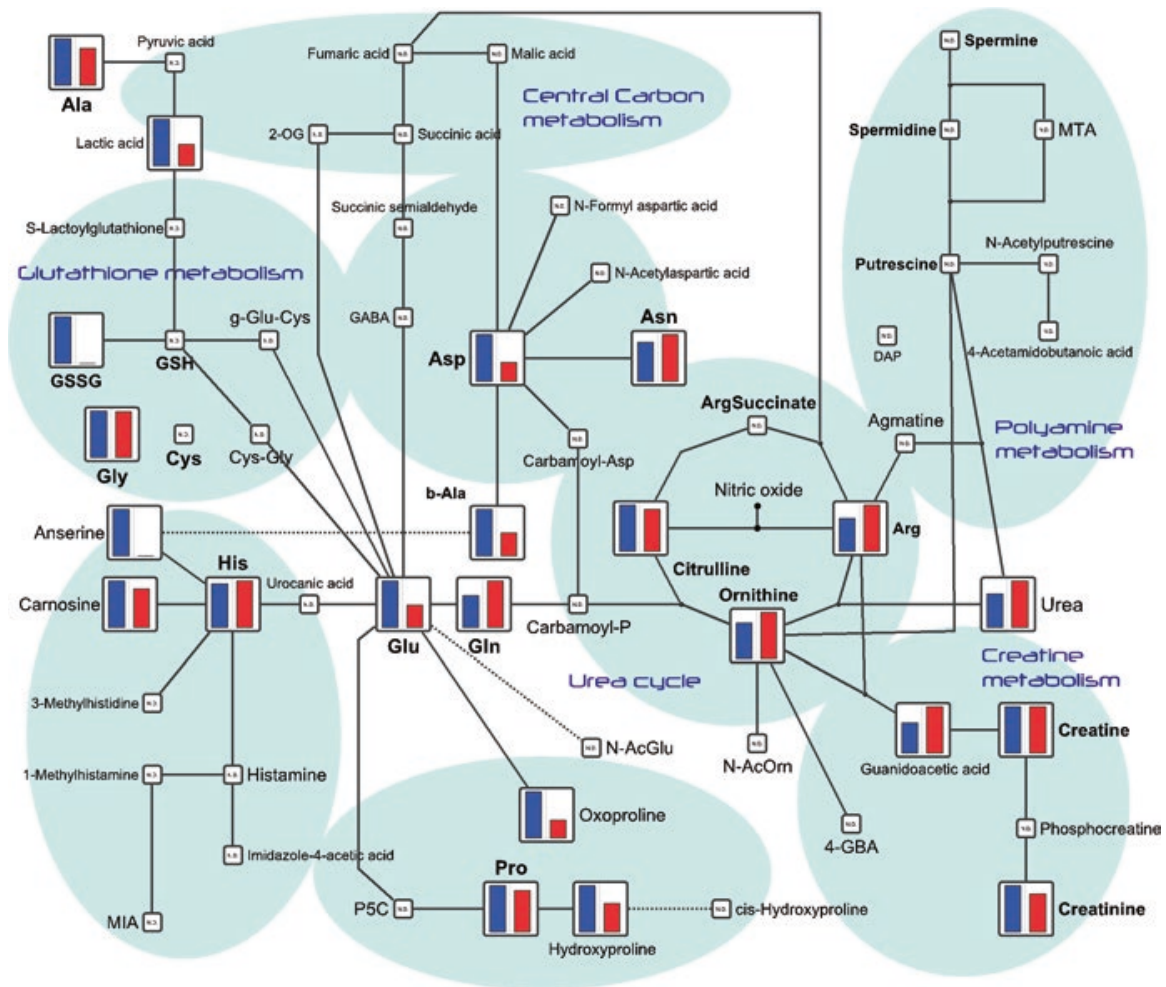

Fig. 10.5 Diagram of urea cycle of the delivered calf (red) and the non-radiocontaminated control (blue). The control relative area was served as the denominator 
Table 10.6 Detected candidate compounds involved in lipid metabolism

\begin{tabular}{l|l|l|l}
\hline \multirow{2}{*}{ Lipid metabolite } & \multicolumn{2}{l|}{ Relative area } & Comparative analysis $^{\mathrm{a}}$ \\
\cline { 2 - 4 } & Control calf & Delivered calf & Ratio \\
\hline Betaine & $4.0 \mathrm{E}-02$ & $4.0 \mathrm{E}-02$ & 1.0 \\
\hline Carnitine & $3.0 \mathrm{E}-03$ & $5.5 \mathrm{E}-03$ & 1.8 \\
\hline Choline & $2.9 \mathrm{E}-02$ & $6.9 \mathrm{E}-03$ & 0.2 \\
\hline Glycerophosphocholine & $1.7 \mathrm{E}-03$ & $3.5 \mathrm{E}-04$ & 0.2 \\
\hline Acetylcarnitine (ALCAR) & $1.3 \mathrm{E}-03$ & $1.5 \mathrm{E}-03$ & 1.2 \\
\hline Phosphorylcholine & $1.2 \mathrm{E}-03$ & $9.1 \mathrm{E}-04$ & 0.7 \\
\hline Sarcosine & $9.6 \mathrm{E}-04$ & $7.6 \mathrm{E}-04$ & 0.8 \\
\hline Taurine & $3.3 \mathrm{E}-03$ & $1.8 \mathrm{E}-03$ & 0.5 \\
\hline
\end{tabular}

${ }^{a}$ Control relative area served as denominator

${ }^{\mathrm{b}}$ Delivered calf associated with paternal exposure to radiation $(n=1)$

urea. Urea levels were not different between the delivered calf and the control (ratio $=1.4)$, as was observed in Arg (ratio = 1.4), citrulline (ratio = 0.9) and ornithine (ratio =1.3). Conversely, Asp showed lower levels in the delivered calf than in the control calf (ratio $=0.4$ ). Although Asp gives a nitrogen atom to urea in the urea cycle, we assume that the difference in Asp levels had no influence on urea production in the delivered calf, since urea levels were not different between them.

Detected candidate compounds associated with lipid metabolism are shown in Table 10.6 and Fig.10.6. Carnitine is a carrier in the mitochondrial membrane responsible for transport of long-chain fatty acids into the mitochondrial matrix, where they are $\beta$-oxidised and used as the energy source in the TCA cycle. Carnitine levels were considerably higher in the delivered calf than in the control (ratio =1.8). 5-Hydroxylysine, acetylcarnitine (ALCAR) and actinin, all associated with carnitine metabolism, were not different between them. Choline is a constituent of phosphatidylcholine and acetylcholine. It is converted to glycerophosphocholine, which is in turn converted to phosphatidylcholine. Glycerophosphocholine serves as a choline source. Choline and glycerophosphocholine were less abundant in the delivered calf than in control calf (ratio $=0.2$ ), suggesting potential shortage in choline supply in the delivered calf as compared with the control calf. Choline is metabolised to betaine, then to sarcosine, and finally to glycine (Gly), but no difference in the levels of those metabolites, as well as that of phosphorylcholine, was observed between them. Taurine plays a role in conjugating bile acids, which are synthesised from cholesterol to form bile salts. Bile salts and acids are discharged from the body in the faeces. Taurine was less abundant in the delivered calf than in the control calf (ratio $=0.5)$, suggesting that bile-salt production tends to be delayed in the delivered calf. 


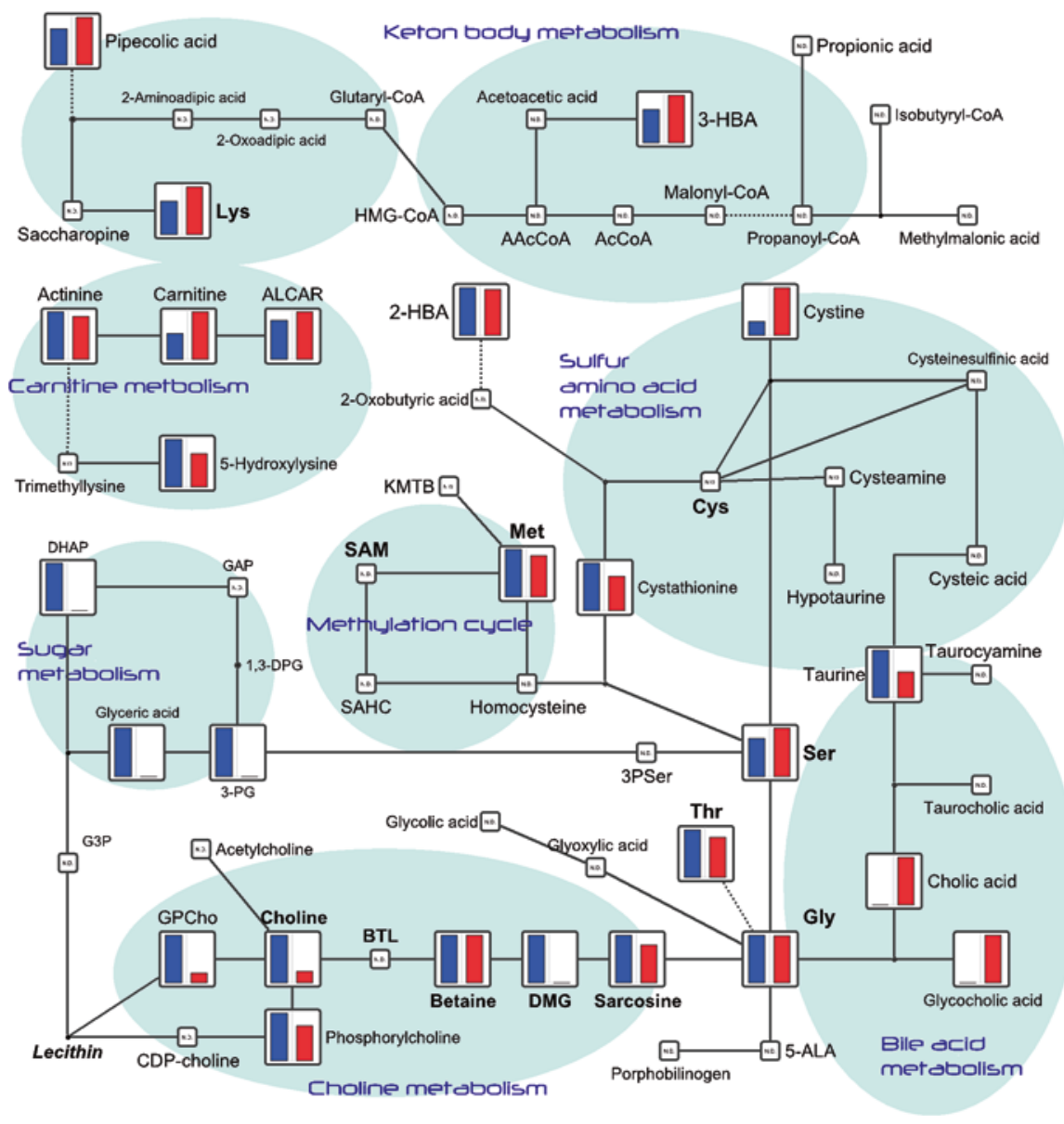

Fig. 10.6 Diagram of lipid metabolism of the delivered calf (red) and non-radiocontaminated control (blue). The control relative area was served as the denominator

\subsection{Conclusions}

In conclusion, there were no transgenerational effects on both of the spermatogenesis and on the blood plasma metabolome in calf obtained by AI using sperm from a LDR-radiated bull in the FNPP ex-evacuation zone. The area of the evacuation zone has been drastically reduced, and no more cattle have been euthanized since February 2014. It is therefore, difficult to investigate more animals with different annual doses. Here, we showed results of only one 13-month-old calf obtained by AI using sperm from a bull exposed to chronic LDR radiation for 2 years in the ex-evacuation zone of the FNPP accident (Fig. 10.1). This study is not a controlled experiment; however, we believe that our results give a framework not only for 
estimating general effects of radiation in cattle but also contributing to the reconstruction of the Fukushima livestock industry and facilitating the improvement of food safety measures.

Acknowledgements We express our gratitude to the Iwaki Livestock Hygiene Service Centre in Fukushima Prefecture, Japan. We thank DVM Yuji Kobayashi and livestock farmers in the 20-km FNPP ex-evacuation zone. We thank DVM Taro Sato at Taro Veterinary Farm, Niigata. This work was partly supported by a grant from the Japan Society for the Promotion of Science (H.Y 15K11952 and M.F. 26253022) and The Programme for Promotion of Basic and Applied Researches for Innovations in Bio-oriented Industry.

Conflict of Interest Statement The authors declare no competing financial interests.

\section{References}

1. Kinoshita N, Sueki K, Kitagawa J et al (2013) Assessment of individual radionuclide distributions from the Fukushima nuclear accident covering central-east Japan. Proc Natl Acad Sci U S A 108:19526-19529

2. Takahashi S, Inoue K, Urushihara Y et al (2015) A comprehensive dose evaluation project concerning animals affected by the Fukushima Daiichi nuclear power plant accident: its setup and progress. J Radiat Res 56(S1):i36-i41

3. Urushihara Y, Kawasumi K, Endo S et al (2016) Analysis of plasma protein concentrations and enzyme activities in cattle within the ex-evacuation zone of the Fukushima Daiichi nuclear plant accident. PLoS One 11:e0155069

4. Koarai K, Kino Y, Takahashi A et al (2016) ${ }^{90} \mathrm{Sr}$ in teeth of cattle abandoned in evacuation zone: record of pollution from the Fukushima-Daiichi nuclear power plant accident. Sci Rep 6:24077

5. Takino S, Yamashiro H, Sugano Y et al (2016) Analysis of the effect of chronic and low-dose radiation exposure on spermatogenic cells of male large Japanese field mice (Apodemus speciosus) after the Fukushima Daiichi nuclear power plant accident. Radiat Res 187:161-168

6. Fukuda T, Kino Y, Abe Y et al (2013) Distribution of artificial radionuclides in abandoned cattle in the evacuation zone of the Fukushima Daiichi nuclear power plant. PLoS One 8:e54312

7. Fukuda T, Kino Y, Abe Y et al (2015) Cesium radioactivity in peripheral blood is linearly correlated to that in skeletal muscle: analyses of cattle within the evacuation zone of the Fukushima Daiichi nuclear power plant. Anim Sci J 86:120-124

8. Fukuda T, Hiji M, Kino Y et al (2016) Software development for estimating the cesium radioactivity in skeletal muscles of cattle from blood samples. Anim Sci J 87:842-847

9. Yamashiro H, Abe Y, Fukuda T et al (2013) Effects of radioactive caesium on bull testes after the Fukushima nuclear plant accident. Sci Rep 3:2850

10. Yamashiro H, Abe Y, Hayashi G et al (2015) Electron probe X-ray microanalysis of boar and inobuta testes after the Fukushima accident. J Radiat Res 56(S1):i42-i47

11. Yamashiro H, Abe Y, Kuwahara Y et al (2014) Cryopreservation of cattle, pig, inobuta sperm and oocyte after the Fukushima nuclear plant accident. In: Recent advances in cryopreservation. IntechOpen, pp 73-81

12. Akiyama M, Takino S, Sugano Y et al (2015) Effect of seasonal changes on testicular morphology and the expression of circadian clock genes in Japanese wood mice (Apodemus speciosus). J Biol Regul Homeostat Agent 29:589-600 
13. Kaneko K, Uematsu E, Takahashi Y et al (2013) Semen collection and polymerase chain reaction-based sex-determination of black-headed and straw-necked ibis. Reprod Domest Anim 48:1001-1005

14. Soga T, Heiger HD (2000) Amino acid analysis by capillary electrophoresis electrospray ionization mass spectrometry. Anal Chem 72:1236-1241

15. Soga T, Ueno Y, Naraoka $\mathrm{H}$ et al (2002) Simultaneous determination of anionic intermediates for Bacillus subtilis metabolic pathways by capillary electrophoresis electrospray ionization mass spectrometry. Anal Chem 74:2233-2239

16. Soga T, Ohashi Y, Ueno Y et al (2003) Quantitative metabolome analysis using capillary electrophoresis mass spectrometry. J Proteome Res 2:488-494

17. Sugimoto M, Wong DT, Hirayama A et al (2010) Capillary electrophoresis mass spectrometrybased saliva metabolomics identified oral, breast and pancreatic cancer-specific profiles. Metabolomics 6:78-95

18. Junker BH, Klukas C, Schreiber F (2006) A system for advanced data analysis and visualization in the context of biological networks. BMC Bioinformatics 7:109

Open Access This chapter is licensed under the terms of the Creative Commons Attribution 4.0 International License (http://creativecommons.org/licenses/by/4.0/), which permits use, sharing, adaptation, distribution and reproduction in any medium or format, as long as you give appropriate credit to the original author(s) and the source, provide a link to the Creative Commons license and indicate if changes were made.

The images or other third party material in this chapter are included in the chapter's Creative Commons license, unless indicated otherwise in a credit line to the material. If material is not included in the chapter's Creative Commons license and your intended use is not permitted by statutory regulation or exceeds the permitted use, you will need to obtain permission directly from the copyright holder.

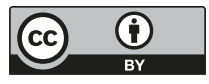

